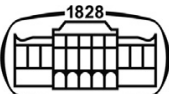

AKADÉMIAI KIADÓ

\section{Acta Veterinaria Hungarica}

68 (2020) 4, 364-369

DOI:

$10.1556 / 004.2020 .00062$

(c) 2021 Akadémiai Kiadó, Budapest

ORIGINAL RESEARCH

PAPER

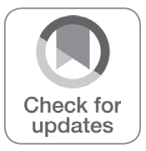

\title{
Effect of gintonin on matrix metalloproteinase-9 concentration in tears during corneal wound healing in rabbits
}

\author{
HA-EUN LEE ${ }^{1}$, JOON-YOUNG KIM ${ }^{1 *} \odot$, \\ SOON-WUK JEONG ${ }^{2}$ and SEUNG-YEOL NAH ${ }^{3}$
}

\author{
${ }^{1}$ Department of Veterinary Ophthalmology, College of Veterinary Medicine, Konkuk University, \\ 120 Neungdong-ro, Gwangjin-gu, Seoul, 05029, Korea \\ ${ }^{2}$ Department of Veterinary Surgery, College of Veterinary Medicine, Konkuk University, Seoul, \\ Korea \\ ${ }^{3}$ Ginsentology Research Laboratory and Department of Physiology, College of Veterinary Medicine, \\ Konkuk University, Seoul, Korea
}

Received: April 9, 2020 • Accepted: December 10, 2020

Published online: February 10, 2021

\begin{abstract}
It has been shown that gintonin, isolated from Panax ginseng, can promote rapid corneal wound healing. We aimed to elucidate the underlying mechanism and investigated whether gintonin affects the concentration of the extracellular matrix remodelling factor matrix metalloproteinase-9 (MMP-9) in tears during rabbit corneal wound healing in vivo. Twelve eyes (six rabbits) were divided equally into three groups. All eyes underwent corneal de-epithelialisation. The control group received Tearin Free sodium hyaluronate $0.1 \%$, the solcoseryl group received solcoseryl- 120 concentrate, and the gintonin group received $2.5 \mathrm{mg}$ gintonin in sodium hyaluronate $0.1 \%$. All preparations were administered for 5 days and the concentration of MMP-9 was measured in tears via ELISA on days 0, 1, and 5. MMP-9 concentrations in all groups were increased at day 1 and reduced at day 5 . Of note, we found a significant change over the time frame for the gintonin group $(P<0.05)$ but not for the control or solcoseryl groups $(P>0.05)$ Moreover, increased MMP-9 levels between days 0 and 1 , and their reduction between days 1 and 5, were significant in the gintonin group compared to those in the other groups $(P<0.05)$; however, and once more, these changes were not significant between the control and solcoseryl groups $(P>0.05)$. In conclusion, gintonin increases the concentration of MMP-9 rapidly within a day of injury, and decreasing it thereafter.
\end{abstract}

\section{KEYWORDS}

corneal epithelial healing, corneal wound healing, gintonin, MMP-9, tear

\section{INTRODUCTION}

Gintonin, isolated from Panax ginseng, contains lysophosphatidic acid (LPA, C18:2) and other LPAs, which activate the G-protein-coupled LPA receptor via high-affinity binding (Hwang et al., 2012). Recently it has been reported that gintonin can promote rapid corneal wound healing in an in vivo rabbit model; of note, in vitro experiments using human corneal epithelial (HCE) cells linked these healing properties to the stimulation of cell migration and division via the $\mathrm{Ca}^{2+}$-signalling pathway by exogenous LPAs in gintonin (Kim et al., 2017). In contrast, gintonin has also been shown to reduce motility and migration of melanoma cells, with a minimal effect on proliferation via strong inhibition of autotaxin activity and LPA production (Hwang et al., 2013). The mechanism underlying the effect of gintonin on corneal wound healing is therefore unclear.

LPA, a well-characterised phospholipid, is known as a lipid-derived growth factor and a mediator of wound healing (Kim et al., 2017). LPA is generated from lysophosphatidylcholine
Corresponding author. Tel.: +82

(02) 450 3720; fax: +82 (02) 450

E-mail: canvet@hanafos.com

E-mail: canvet@konkuk.ac.kr 
(LPC) via autotaxin (ATX), with lysophospholipase D activity (lysoPLD), which hydrolyses the choline from the phosphate head group (Benesch et al., 2016). LPA binds to the specific G-protein-coupled LPA receptor and evokes diverse downstream cell signalling pathways to induce cell proliferation and migration, and production of cytokines and matrix metalloproteinases (MMPs) (Van Meeteren and Moolenaar, 2007; Pua et al., 2009). MMPs are a family of extracellular endoproteinases that degrade extracellular matrix proteins such as laminin, fibronectin, and collagen (Li et al., 2001). The main functions of MMPs in normal corneal physiology are the degradation and remodelling of the stroma and the control of epithelial basement membrane synthesis (Chow et al., 2015). In the normal cornea, the digestive properties of these enzymes are antagonised by the tissue inhibitors of metalloproteinases (TIMPs); however, when an imbalance develops between MMPs and TIMPs, disintegration or digestion of the corneal extracellular matrix occurs, leading to keratomalacia (Chow et al., 2015). Two gelatinases, MMP-2 and MMP-9, are the primary matrixdegrading enzymes produced by the corneal epithelium (Li et al., 2001). In most species, MMP-2 is constitutively present in unwounded corneal epithelium and stroma and is upregulated after wounding, whereas MMP-9 is found only in wounded corneas (Ledbetter and Gilger, 2013). MMP-9 is involved in the early stages of corneal epithelial wound healing, and MMP-2 may be important in extracellular matrix remodelling in the later stages of corneal wound healing (Ye and Azar, 1998). Recent studies have shown that damaged corneas exhibit increased secretion of LPA, which could be involved in wound healing (Kim et al., 2017). Importantly, there is an inhibitory mechanism that regulates the production of MMP-9 to prevent its overproduction as a result of increased LPA after corneal injury. In the steadystate, the concentration of LPA in plasma is low because there is a tightly controlled balance between its production and degradation. The suggested mechanism for the maintenance of this balance is LPA-mediated negative feedback loop via ATX (Van Meeteren and Moolenaar, 2007). This said, a drug inducing the faster onset of corneal healing could expedite healing and allow shorter treatment courses.

The purpose of this study was to determine whether exogenous LPAs in gintonin affect the concentration of MMP-9 in tears during rabbit corneal wound healing in vivo.

\section{MATERIALS AND METHODS}

\section{Animals}

Six female 9- to 11-week-old New Zealand white rabbits weighing $2.0-2.5 \mathrm{~kg}$ were obtained from a commercial supplier. Rabbits were fed a commercial pelleted diet provided with water ad libitum. The experimental environment was managed under standard conditions: the rabbits were floor housed one per cage, at $23 \pm 1{ }^{\circ} \mathrm{C}$ with $55 \pm 5 \%$ humidity, under 12-h light-dark cycles (6 AM-6 PM). All procedures using animals were approved by the Institutional Animal Care and Use Committee (IACUC) of Konkuk University (permit No. KU172048).

\section{Study procedure}

The rabbits were divided into three treatment groups $(n=2$ per group; each rabbit was given the same treatment in both eyes) to evaluate MMP-9 concentration in tears: control group (sodium hyaluronate $0.1 \%$, Tearin Free; DHP Korea, Cheongju, Korea), solcoseryl (solcoseryl 120 concentrate, Solcorin; Hanlim Pharmaceuticals, Seoul, Korea), and gintonin (2.5 mg gintonin with sodium hyaluronate $0.1 \%$, Eyelein; Unimed Pharm Inc., Seoul, Korea) treatment groups.

\section{Surgical procedure}

The corneal wound was induced under general anaesthesia. Sedation was achieved with medetomidine via intramuscular injection $(0.35 \mathrm{mg} / \mathrm{kg})$. Induction and maintenance were carried out using a face mask (base $6.35 \mathrm{~cm}$, hole diameter $3.3 \mathrm{~cm}$, length $4 \mathrm{~cm}$ ) (mask; VetEquip Inc., US Item Number: 921417) providing a continuous flow of isoflurane at $1-2 \%$. Of note, isoflurane was delivered in increased concentrations of anaesthetic vapour, beginning at $2 \%$, with $0.5 \%$ increments every $15-30 \mathrm{~s}$ to a maximum of $5 \%$. Anaesthetic monitoring included electrocardiogram, heart rate, systolic, diastolic and mean blood pressure, rectal temperature, and arterial haemoglobin oxygen saturation (SpO2) evaluation.

Before we induced the corneal wound, local anaesthetic eye drops (proparacaine, Alcaine; Alcon, Geneva, Switzerland) and topical antibiotic eye drops (ofloxacin, Tarivid, Santen, Osaka, Japan) had been administered to all rabbits. Corneas were irrigated with $20 \mathrm{~mL}$ of $0.2 \%$ povidoneiodine solution for ocular surface disinfection. A 6-mm biopsy punch (Kai Medical, Japan) was used to make a shallow guide mark at the centre of the cornea. A 70\% alcohol-soaked cotton swab was applied to the cornea very carefully to avoid injury outside of the guide mark. The epithelial layer was removed with a corneal epithelial burr (Algerbrushll; Alger, New York, NY, USA). A fluorescein test (Fluorets; Chauvin, Paris, France) was performed to confirm that the full epithelial layer of the cornea was damaged. After surgery, artificial tears (sodium hyaluronate $0.1 \%$, Tearin Free; DHP Korea), solcoseryl (solcoseryl 120 concentrate, Solcorin; Hanlim Pharmaceuticals), and gintonin (2.5 mg gintonin with sodium hyaluronate $0.1 \%$ ) (sodium hyaluronate $0.1 \%$, Eyelein, Unimed) eye drops were applied to the control, solcoseryl, and gintonin groups, respectively, every $8 \mathrm{~h}$. Topical antibiotic eye drops were also applied every $8 \mathrm{~h}$ to all groups.

\section{Tear collection and determination of tear MMP-9 concentration using an enzyme-linked immunosorbent assay (ELISA) kit}

The schedule of tear collection was set up as per the degree of corneal re-epithelialisation using a fluorescein staining test. 
The fluorescence staining area was recorded using an $8 \times$ slitlamp microscope (Hawk Eye; Dioptrix, Toulouse, France). Because corneas of all groups no longer stained with fluorescein on day 5 , we performed tear collection on days 0,1 , and 5. All procedures were carried out under general anaesthesia. At each collection, tears were taken three times per eye from the lower (inferior) conjunctival fornix using a cellulose eye spear sponge (Eyelike Eye Spear BS40400, KORYO EyeTech, Dhaka, Bangladesh). Polypropylene microcentrifuge tubes (SPL Life Sciences, Gyeonggi-do, Korea) were prepared by making a $1.20 \mathrm{~mm}$ hole at the bottom of the tube. The sponge part of the eye spear absorbing the tears was cut and placed into the tube, which was then put into a $15-\mathrm{mL}$ conical centrifuge tube (SPL Life Sciences). Centrifugation (Hanil Biomed) was carried out at $4,000 \mathrm{rpm}$ for $20 \mathrm{~min}$ to collect the tear samples, which were immediately transferred to another polypropylene microcentrifuge tube and stored at $-80{ }^{\circ} \mathrm{C}$ until analysis.

The MMP-9 content of each tear sample was determined via ELISA according to the manufacturer's protocol (Rabbit Matrix Metalloproteinase 9 Elisa Kit, Competitive ELISA; MyBioSource Inc., San Diego, CA, USA). Optical density (O.D.) values were measured using a microplate spectrophotometer (Multiskan ${ }^{\mathrm{TM}}$ FC, Thermo Fisher Scientific, Waltham, MA, USA) at $450 \mathrm{~nm}$.

\section{Statistical analysis}

To verify the MMP-9 content changes for all groups, we calculated the differential amount of MMP-9 as follows:

$$
\begin{aligned}
\mathrm{P} 1= & \text { MMP-9 concentration at day } 1 \\
& - \text { MMP-9 concentration at day } 0 \\
\mathrm{P} 2= & \text { MMP-9 concentration at day } 5 \\
& - \text { MMP-9 concentration at day } 1
\end{aligned}
$$

We compared the MMP-9 concentrations on days 0,1 , and 5 among groups, as well as P1 and P2 values from all groups, using the Kruskal-Wallis test. Subsequently, pairwise differences between the P1 and P2 values of the groups were analysed using the one-tailed Mann-Whitney $U$ test as a post hoc analysis. For each test, $P<0.05$ was considered significant. Statistical tests were performed with the SPSS software (ver. 22; IBM, Armonk, NY, USA).

\section{RESULTS}

Representative results of the fluorescein dye test performed 0 , 1 , and 5 days after surgery are shown in Fig. 1 . All corneas in all groups stained positively at day 1 and did not stain at day

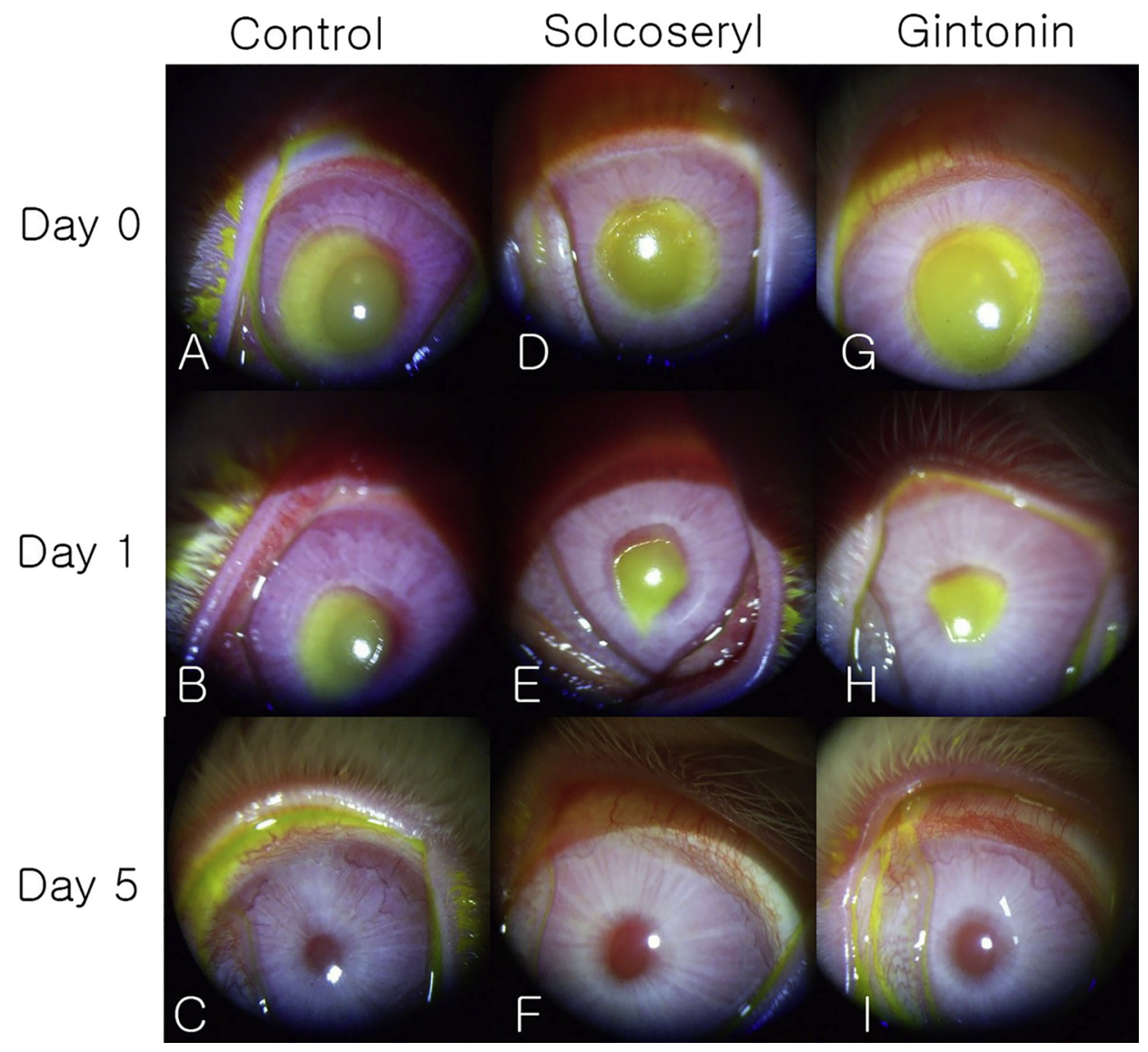

Fig. 1. Representative results of the fluorescein dye test 0,1 , and 5 days after surgery (A, B, C: control group; D, E, F: solcoseryl group; G, H, I: gintonin group). In all groups, the cornea was stained with the fluorescent dye, one day after corneal injury. All corneas were completely healed on day 5 


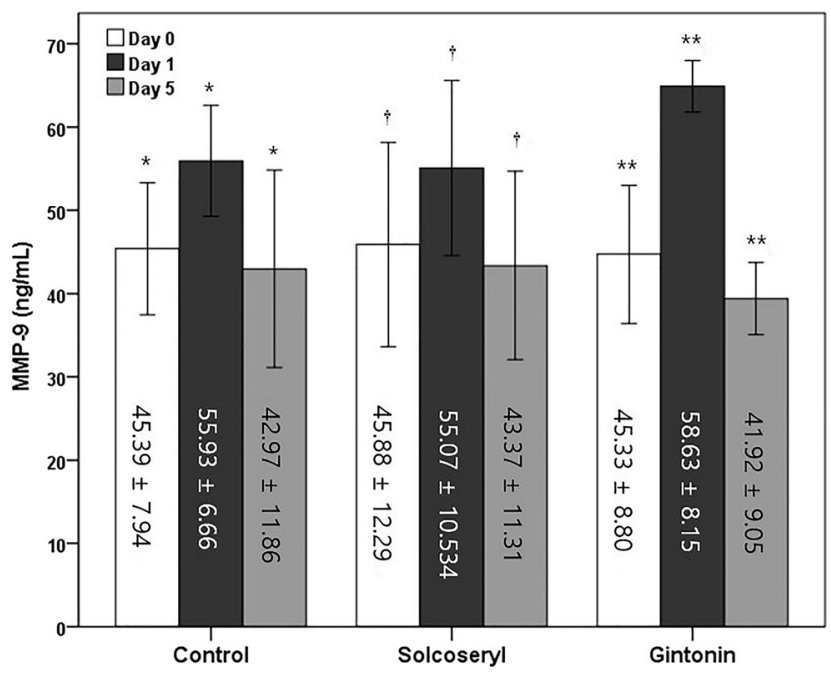

Fig. 2. Matrix metalloproteinase-9 (MMP-9) concentration 0, 1, and 5 days after surgery. Comparison of MMP- 9 concentration on days 0,1 , and 5 in ${ }^{*}$ the control group $(P=0.232)$, $\dagger$ the solcoseryl group $(P=0.232)$, and ${ }^{\star *}$ the gintonin group $(P=0.015)$ (KruskalWallis test)

5 , suggesting that they were completely healed. The changes in MMP-9 concentration from day 0 to days 1 and 5 in the three groups are shown in Fig. 2. MMP-9 concentration increased in all groups at day 1 and decreased at day 5 . Comparing these concentrations statistically, we found a significant change over the time for the gintonin group $(P=$ $0.015)$ but not for the control or solcoseryl groups $(P=0.232$ and $P=0.232$, respectively). Breaking this down further, we examined MMP-9 concentration changes between days 0 and 1 , and days 1 and 5 (P1 and P2, respectively; Table 1). P1 and $\mathrm{P} 2$ were significantly different among the three groups $(P=$ 0.023 and $P=0.024$, respectively, Table 1 ). Moreover, pairwise comparisons revealed that the $\mathrm{P} 1$ value of the gintonin group was significantly higher than those of the control or solcoseryl groups ( $P=0.029$ and $P=0.029$, respectively).

Table 1. Differential amount of matrix metalloproteinase-9 (MMP-9) between two time-points, per group (mean \pm SD)

\begin{tabular}{lrrrr}
\hline & \multicolumn{1}{c}{ Control } & \multicolumn{1}{c}{ Solcoseryl } & Gintonin & \multicolumn{2}{c}{$P$ value $^{\mathrm{a}}$} \\
\hline $\mathrm{P}^{\mathrm{b}}(\mathrm{ng} / \mathrm{mL})$ & $10.55 \pm 1.61$ & $9.18 \pm 2.87$ & $20.18 \pm 6.63$ & 0.023 \\
$\mathrm{P}^{\mathrm{c}}(\mathrm{ng} / \mathrm{mL})$ & $-12.96 \pm 5.76$ & $-11.69 \pm 2.76$ & $-25.48 \pm 2.75$ & 0.024 \\
\hline
\end{tabular}

As per pairwise comparisons, the P1 value of the gintonin group was significantly higher than those of the control and solcoseryl groups ( $P=0.029$ and $P=0.029$, respectively). Additionally, the $\mathrm{P} 2$ value of the gintonin group was significantly lower than those of the control and solcoseryl groups $(P=0.029$ and $P=0.029$, respectively; one-tailed Mann-Whitney $U$ test). However, the P1 and $\mathrm{P} 2$ values were not significantly different between the control and solcoseryl groups $(P=0.686$ and $P=0.886$, respectively; onetailed Mann-Whitney $U$ test).

${ }^{\mathrm{a}}$ Kruskal-Wallis test.

${ }^{\mathrm{b}} \mathrm{P} 1=$ MMP-9 concentration at day $1-$ MMP-9 concentration at day 0.

${ }^{c} \mathrm{P} 2=$ MMP-9 concentration at day $5-$ MMP-9 concentration at day 1 .
Additionally, the P2 value of the gintonin group was significantly lower than those of the control and solcoseryl groups ( $P=0.029$ and $P=0.029$, respectively). Of note, the $\mathrm{P} 1$ and P2 values were not significantly different between the control and solcoseryl groups $(P=0.686$ and $P=0.886$, respectively). Therefore, the increase in MMP-9 between days 0 and 1 , and its reduction between days 1 and 5, were significant in the gintonin group compared to the other groups $(P<0.05)$.

\section{DISCUSSION}

In this study, we show that the increase of MMP-9 from day 0 to day 1 after corneal lesion in the gintonin group is significantly different from that of the other groups. Moreover, we also show that the reduction of MMP-9 from day 1 to day 5 in the gintonin group is significantly different than that in the other groups in the context of corneal wound healing. Gintonin has unique protein components and a high concentration of LPA (Im and Nah, 2013). When corneal epithelial damage occurs, autotaxin (ATX) is increased by various cytokines, and promotes the upregulation of LPA. LPA binds to the G-protein-coupled LPA receptor to produce various wound-healing effects and MMP-9 expression (Benesch et al., 2016). This was reflected in our data, as in all three groups the early stage of corneal wound healing was associated with a short-term increase in MMP-9 concentration, although this was not significant in the control or solcoseryl groups. Because gintonin is composed of LPA, it probably promotes the production of MMP-9. This is the obvious explanation for the significant increase of MMP-9 levels in the gintonin group during the first day of healing. It has previously been shown that the pattern of MMP-9 synthesis is consistent with the timing of basement membrane degradation; there is a rapid increase in MMP-9 expression within a day of wounding, followed by a loss of expression a few weeks afterwards (Sivak and Fini, 2002). In line with this, in the present study, gintonin significantly increased the concentration of MMP-9 within a day of wounding and significantly decreased it afterwards.

How does gintonin significantly decrease MMP-9 from days 1 to 5 after corneal lesion compared to the other groups? One possibility is that the LPA in gintonin can suppress the activity of ATX via the enhancement of a negative feedback loop. A recent report has shown that LPA C18:2 inhibits ATX activity more than the other LPAs (Im and Nah, 2013). Of note, the order of LPA species in gintonin from the most to the least abundant is LPA C18:2 > LPA C16:0 > LPA C18:1 (Kim et al., 2018). Maximal inhibition of ATX is induced by long-chain LPAs (18:1, 16:0, and 14:0), whereas short-chain LPA (6:0) has no effect on ATX (Van Meeteren and Moolenaar, 2007). The requirement for a long acyl chain suggests that LPA directly interacts with a hydrophobic pocket on the ATX molecule (Van Meeteren and Moolenaar, 2007). Therefore, the results of this study are consistent with the inhibition of ATX via the binding of LPA C18:2 to its hydrophobic pocket. 
We should also consider the role of gintonin protein components in the regulation of MMP-9 by gintonin. The LPAs in gintonin exist as complexes with ginseng proteins (Im and Nah, 2013). The protein components of gintonin may play three roles that are related to LPA activities: (1) they may function as LPA carriers or LPA storage molecules; (2) they may lessen the access of LPA hydrolysing enzymes; (3) they may provide a hydrophobic environment that enables efficient delivery of LPAs to their cognate receptors (Im and Nah, 2013). Therefore, the protein components of gintonin may function as carriers and/or stabilisers of LPAs and may help LPAs act as functional, high-affinity ligands of G-protein-coupled LPA receptors (Kim et al., 2017). However, the mechanisms of action of LPAs and ginseng proteins are not yet clear and further studies are needed.

However, this study is not without limitations. First, we did not measure the MMP-9 concentration daily. The measurement of the concentration of MMP-9 in tears on a daily basis would have allowed us to understand better the dynamics of MMP-9 concentration during corneal wound healing. However, for this we would have had to carry out anaesthesia of rabbits every day to collect tears. Anaesthetic procedures can cause stress in rabbits, and thus animal welfare was one of the reasons why we did not do MMP-9 measurements on a daily basis. Moreover, we thought that anaesthetic procedures would result in consequent pain and stress, which could also affect corneal healing (potentially compromising accurate conclusions). Therefore, instead of tear collection every day, we thought that the change in MMP-9 concentration and the degree of corneal re-epithelialisation would be highly related. So, the schedule of tear collection was determined by evaluating the degree of corneal re-epithelialisation. So, we measured the concentration of MMP-9 in tears on the day of inducing the corneal lesion and then one day and five days thereafter, when epithelial regeneration was complete.

Second, the objective numerical measurement of corneal depth after de-epithelialisation was not performed. The quantification of de-epithelialisation would have been essential to support the repeatability and reproducibility of the deepithelialisation technique used. However, we were not able to adopt objective corneal thickness measurement due to technical limitations. This said, we applied alcohol to devitalise only the corneal epithelium, and used an epithelial burr (Algerbrush II) to remove only the epithelium. Moreover, to potentially increase the reproducibility of the surgical intervention, the same investigator carried out the whole procedure in all animals. Still, in the future, we will include the measurement of corneal depth in our experimental layout to obtain more reliable data (and conclusions).

Third, because of the relatively small sample size, both eyes of each rabbit were treated with the same drug in the present study. This experimental design does not adequately account for variability in the healing behaviours of individual rabbits, which may have influenced the MMP-9 ELISA findings. Of note, in a previous study, we used the same experimental layout in rabbits (Kim et al., 2017). We applied the same drug preparation to both eyes of each rabbit. We designed the experimental groups this way because we intended to minimise the use of experimental animals. Moreover, we thought that the amount of gintonin used was too small to lead to systemic absorption; therefore, the local administration on one eye will not affect the other eye. However, the application of gintonin to one eye and placebo to the other would have probably allowed us to obtain more reliable results, a strategy we plan to follow in subsequent studies.

Fourth, we only compared gintonin with solcoseryl (and did not include other positive controls). If we could have compared the effects of other medications to treat corneal wound healing with those caused by gintonin, our results would have been more complete. However, solcoseryl is a component extracted from the blood of calves and is widely used for the treatment of cornea and conjunctiva (and therefore is a relevant positive control). Importantly in the case of solcoseryl, there are already published studies on its influence on the expression MMP-9 and MMP-2, which allow a direct comparison (Chow et al., 2015; Nam and Maeng, 2019). Therefore, we used solcoseryl as a representative corneal wound healing medicine affecting MMP-9, to contextualise the effects of gintonin.

MMP-9 produced at the beginning of healing induces epithelial cell migration by degrading the extracellular matrix. As such, although MMP-9 aids in corneal epithelialisation at the early stage, opposite effects appear to occur in the middle and later stages. If the reduction of MMP-9 concentration does not proceed in the middle and later stages effectively, excessive degrading of the extracellular matrix causes delayed corneal healing. As corneal healing is delayed, the possibility of corneal fibrosis increases, which can reduce corneal clarity. Gintonin acts to modulate MMP9 concentration with two opposing mechanisms during the corneal wound healing process. Therefore, if gintonin eye drops are clinically used to control MMP-9 concentration, this will result in a good and rapid therapeutic effect.

In summary, gintonin increased the concentration of MMP-9 rapidly within a day after corneal injury and decreased the concentration of MMP-9 afterwards. In conjunction with the results of previous works, the data in our study suggest that gintonin modulates MMP-9 expression via two opposing mechanisms during the process of corneal wound healing. With these mechanisms, gintonin eye drops can aid rapid corneal wound healing, resulting in a good therapeutic effect.

\section{REFERENCES}

Benesch, M. G., Tang, X., Venkatraman, G., Bekele, R. T. and Brindley, D. N. (2016): Recent advances in targeting the autotaxin-lysophosphatidate-lipid phosphate phosphatase axis in vivo. J. Biomed. Res. 30, 272-284.

Chow, D. W., Chau, Y., Yeung, W. K. and Westermeyer, H. D. (2015): In vitro evaluation of the inhibitory effect of canine 
serum, canine fresh frozen plasma, freeze-thaw-cycled plasma, and Solcoseryl on matrix metalloproteinases 2 and 9. Vet. Ophthalmol. 18, 229-233.

Hwang, S. H., Lee, B. H., Kim, H. J., Cho, H. J., Shin, H. C., Im, K. S., Choi, S. H., Shin, T. J., Lee, S. M., Nam, S. W., Kim, H. C., Rhim, H. and Nah, S. Y. (2013): Suppression of metastasis of intravenously-inoculated B16/F10 melanoma cells by the novel ginseng-derived ingredient, gintonin: involvement of autotaxin inhibition. Int. J. Oncol. 42, 317-326.

Hwang, S. H., Shin, T. J., Choi, S. H., Cho, H. J., Lee, B. H., Pyo, M. K., Lee, J. H., Kang, J., Kim, H. J., Park, C. W., Shin, H. C. and Nah, S. Y. (2012): Gintonin, newly identified compounds from ginseng, is novel lysophosphatidic acids-protein complexes and activates G protein-coupled lysophosphatidic acid receptors with high affinity. Mol. Cells 33, 151-162.

Im, D. S. and Nah, S. Y. (2013): Yin and Yang of ginseng pharmacology: ginsenosides vs. gintonin. Acta Pharmacol. Sin. 34, 1367-1373.

Kim, H. J., Kim, J. Y., Lee, B. H., Choi, S. H., Rhim, H., Kim, H. C., Ahn, S. Y., Jeong, S. W., Jang, M., Cho, I. H. and Nah, S. Y. (2017): Gintonin, an exogenous ginseng-derived LPA receptor ligand, promotes corneal wound healing. J. Vet. Sci. 18, 387397.

Kim, H. J., Jung, S. W., Kim, S. Y., Cho, I.-H., Kim, H. C., Rhim, H., Kim, M. and Nah, S. Y. (2018): Panax ginseng as an adjuvant treatment for Alzheimer's disease. J. Ginseng Res. 42, 401-411.

Ledbetter, E. C. and Gilger, B. (2013): Diseases and surgery of the canine cornea and sclera. In: Gelatt, K. N., Gilger, B. C. and Kern, T. J. (eds) Veterinary Ophthalmology. Fifth Edition. Wiley-Blackwell, John Wiley \& Sons, Inc., Ames, Iowa, USA. pp. 976-1049.

Li, D. Q., Lokeshwar, B. L., Solomon, A., Monroy, D., Ji, Z. and Pflugfelder, S. C. (2001): Regulation of MMP-9 production by human corneal epithelial cells. Exp. Eye Res. 73, 449-459.

Nam, S. M. and Maeng, Y. S. (2019): Wound healing and mucin gene expression of human corneal epithelial cells treated with deproteinized extract of calf blood. Curr. Eye Res. 44, 1181-1188.

Pua, T. L., Wang, F.-Q. and Fishman, D. A. (2009): Roles of LPA in ovarian cancer development and progression. Future Oncol. 5, 1659-1673.

Sivak, J. M. and Fini, M. E. (2002): MMPs in the eye: emerging roles for matrix metalloproteinases in ocular physiology. Prog. Retin. Eye Res. 21, 1-14.

Van Meeteren, L. A. and Moolenaar, W. H. (2007): Regulation and biological activities of the autotaxin-LPA axis. Prog. Lipid Res. 46, 145-160.

Ye, H. Q. and Azar, D. T. (1998): Expression of gelatinases A and B, and TIMPs 1 and 2 during corneal wound healing. Invest. Ophthalmol. Vis. Sci. 39, 913-921. 